\title{
GROWTH RATE OF GAUSSIAN PROCESSES WITH STATIONARY INCREMENTS
}

\author{
BY STEVEN OREY
}

Communicated by David Blackwell, November 3, 1969

1. Statement of results. Let $(\mathrm{Y}, t, t \geqq 0)$ be a real, separable Gaussian process with stationary increments, mean 0 , and $Y_{0}=0$. Let $2 Q(t)$ be the variance of $Y_{t}$ and define

$$
X_{t}=Y_{t} /(2 Q(t))^{1 / 2} \text {. }
$$

THEOREM 1. Suppose there exists a nonnegative function $v(t)$ such that

$$
\lim _{t \rightarrow \infty} \frac{Q(s+t)-Q(s)}{v(s+t)-v(s)}=1 \text { uniformly in } s
$$

and there exist positive constants $s_{0}, \beta_{1}, \beta_{3}$ with $1 \leqq \beta_{3} \leqq\left(\beta_{1} / 2+1\right)$ such that

(i) is monotone nondecreasing,

(ii) $v(\lambda s) \geqq \lambda^{\beta_{1}} v(s)>0, \quad s \geqq s_{0}, \lambda \geqq 1$,

(iii) $v(\lambda s) \leqq \lambda^{\beta_{s}} v(s), \quad s \geqq s_{0}, \lambda \geqq 1$

and suppose that there exists $\beta_{2}>0$ such that

(iv) $Q(t)=O\left(t^{\beta_{2}}\right), \quad t \downarrow 0$.

Then

$$
\limsup _{t \rightarrow \infty}\left(X_{t}-(2 \log \log t)^{1 / 2}\right)=0 \quad \text { a.s. }
$$

In fact somewhat more is true.

THEOREM 2. Under the assumptions of Theorem 1,

$$
\lim _{T \rightarrow \infty}\left(\sup _{t \leqq T} X_{t}-(2 \log \log T)^{1 / 2}\right)=0 \quad \text { a.s. }
$$

An important class of examples is obtained by taking $Y_{t}=\int_{0}^{t} Y_{s}^{\prime} d s$ where $\left(Y_{s}^{\prime}\right)$ is a real stationary Gaussian process with mean 0 and continuous sample functions. If $q(|t-s|)$ is the covariance of the $\left(Y_{t}\right)$-process and $R(t)=\int_{0}^{t}\left(q(s) d s\right.$ then $Q(t)=\int_{0}^{t} R(s) d s$. If $v(t)$ is a differentiable function satisfying conditions (i), (ii) and (iii) of The-

AMS Subject Classifications. Primary 6030, 6069.

Key Words and Phrases. Gaussian processes, stationary increments, law of the iterated logarithm, limiting distribution of the maximum. 
orem 1 and $R(t) \sim v^{\prime}(t)$ as $t \rightarrow \infty$, then (*) will hold, and condition (iv) will hold with $\beta_{2}=2$. In particular if $R(t)$ tends to a positive constant $K$, the hypotheses of the theorem will be satisfied with $v(t)=K t$. This will be the case if $q(t)$ is always nonnegative and tends to zero sufficiently rapidly as $t$ approaches infinity, e.g. when $\left(Y_{t}^{\prime}\right)$ is the stationary Ornstein-Uhlenback process with covariance $e^{-|t-s|}$.

2. Idea of proof. Note that the covariance of $\left(Y_{t}\right)$, hence that of $\left(X_{t}\right)$ depends only on the function $Q$. In fact

$$
E\left[Y_{s} Y_{t}\right]=Q(s)+Q(t)-Q(t-s) .
$$

The results are reduced to the following theorem on stationary Gaussian processes; the proof is given in [1].

Theorem (PICKANDs). Let $\left(Z_{t}, t \geqq 0\right)$ be a real, separable, stationary Gaussian process, with mean 0 and covariance $r(|t-s|)$ such that for some $\alpha>0$,

$$
1-r(t)=O\left(t^{\alpha}\right), \quad t \downarrow 0
$$

and

$$
r(t)=O\left((\log t)^{-1}\right), \quad t \rightarrow \infty .
$$

Then

$$
\lim _{T \rightarrow \infty}\left(\sup _{t \leqq T} Z_{t}-(2 \log T)^{1 / 2}\right)=0 .
$$

The plan is to compare $X_{\imath}^{*}=X_{e^{t}}$ with stationary processes to which the theorem of Pickands is applicable. In [2] the following important result is proved.

Lemma (Slepian). Let $X_{j}^{-}, X_{j}^{+}, j=1,2 \cdots N$ be two Gaussian sequences of random variables, with mean 0 and covariances $r-(i, j)$, $r^{+}(i, j)$ respectively, $1 \leqq i \leqq N, 1 \leqq j \leqq N$. Suppose

$r^{-}(i, i)=r^{+}(i, i), i=1,2 \cdots N ; \quad r^{-}(i, j) \leqq r^{+}(i, j), 1 \leqq i \leqq j \leqq N$.

Then for any choice of constants $a_{j}, j=1,2, \cdots N$

$$
P\left[\bigcap_{j=1}^{N}\left[X_{j}^{-} \leqq a_{j}\right]\right] \leqq P\left[\bigcap_{j=1}^{N}\left[X_{j}^{+} \leqq a_{j}\right]\right] .
$$

The argument essentially consists of showing that the hypotheses of Theorem 1 permit construction of stationary processes $\left(X_{t}^{-}\right),\left(X_{t}^{+}\right)$ satisfying the conditions of the theorem of Pickands and having covariances related to that of $\left(X_{t}^{*}\right)$ in such a manner that Slepian's 
lemma allows one to deduce that $\left(X_{t}^{*}\right)$ also satisfies the conclusion of Pickands's theorem.

\section{REFERENCES}

1. J. Pickands III, Maxima of stationary Gaussian processes, Z. Wahrscheinlichkeitstheorie und Verw. Gebeite 7 (1967), 190-223. MR 36 \#955.

2. D. Slepian, The one-sided barrier problem for Gaussian noise, Bell System Tech. J. 41 (1962), 463-501. MR 24 \#A3017.

University of Minnesota, Minneapolis, Minnesota 55455 